\title{
Internet of things based vital signs monitoring system: A prototype validity test
}

\author{
Osman Yakubu, Emmanuel Wereko \\ Department of Computer Science, Garden City University College, Kumasi, Ghana
}

\begin{tabular}{l} 
Article Info \\
\hline Article history: \\
Received Feb 22, 2021 \\
Revised May 20, 2021 \\
Accepted Jun 1, 2021 \\
\hline
\end{tabular}

Keywords:

Body temperature

Heart rate

Internet of Things

Microcontroller

Respiration rate

\begin{abstract}
The advent of the internet of things (IoT) has resulted in an upsurge in the deployment of digital healthcare systems enabling patients' health conditions to be remotely monitored. This article presents an intelligent and automated IoT-based vital signs monitoring system to aid in patient care. A theoretical framework was established to guide the development of a prototype. It encompasses the patient, IoT sensors, input and storage unit, and data processing, analysis and data transmission. The prototype is equipped with the capability of sensing a patient's body temperature, heart rate, and respiration rate in real time and transmits the data to a cloud data repository for storage and analysis. Alerts are sent to caregivers using SMS, email, and voice calls where urgent attention is required for the patient. The voice call is to ensure a caregiver does not miss the alert since SMS and email may not be checked on time. To ensure privacy of patients, a caregiver has to be biometrically verified by either fingerprint or facial pattern. The experimental results confirmed the accuracy of the data gathered by the prototype, privacy of patients is also guaranteed compared to other benchmark systems.
\end{abstract}

This is an open access article under the CC BY-SA license.

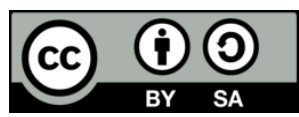

\section{Corresponding Author:}

Osman Yakubu

Department of Computer Science

Garden City University College

P.O. Box KS 12775, Kumasi, Ghana

Email: osman.yakubu@gcuc.edu.gh

\section{INTRODUCTION}

Global economies have been advancing at a rapid pace and there is also the growth of the aging sector of the population and chronic diseases have also been prevalent across the world [1]. This thus presents an urgent need to find and deploy innovative ways for the improvement of patient care, increasing access to health care and reducing the cost of medical care for the benefit of the population. The current innovations in sensor technology, the global accessibility of cellular technology and inexpensive and falling prices of communication devices that are embedded, are opening up innovative channels for the improvement of patient care and the enhancement of quality of life [2]. The internet of things (IoT) is the latest technology that has transformed the traditional computing landscape in all spheres including health care. It is a system that encompasses interconnected computing devices, digital equipment, animals or people that are provided with identifiers that are unique [3]. The IoT is equipped with the capability of transferring data seamlessly to cloud locations for storage and analysis. In the estimation of Ahmed et al. [4], the usage of remote monitoring systems on a larger scale has been hampered by a number of issues such as power consumption, security, and data exchange efficiency, giving an edge to IoT deployment. The IoT which currently spurs the healthcare domain plays a very significant role in varied health care monitoring applications [5]. Sensor 
devices that are networked and are either worn on the body or embedded in living environments [6] has made it possible to gather rich information for the evaluation of the health condition of patients through the collection of data on body temperature, blood pressure and sugar level amongst others. Ways of communicating the collected patient information to a caregiver, giving accurate decision on the gathered data and sending a notification to the patient continues to be a challenging task in the IoT domain. Iyer and Mishra [7] posit that with the advent of the IoT, it is estimated by market analysts that almost 25 billion IoT devices would be connected worldwide by 2020 and the market for IoT would expand to about 2.1 trillion by 2025 . One of the sectors that the IoT is currently thriving is the health sector. Forbes magazine reports that the IoT market within the health industry is expected to exceed $\$ 117$ billion by 2020 [8]. In the medical industry, the IoT could produce various applications that will culminate in the improvement of quality of life, safeguard lives, and curtail cost of treatment. Errors in recording patients' vital sign can also be greatly minimized. With the current deployment of high and advanced sensor technology, activities of human and other objects is easier to track, researchers therefore must commence the discovery of technological solutions that will improve the health care system [9]. This article therefore aims to present an appropriate IoT-based system that monitors vital signs: body temperature, heart rate, and respiratory rate which will be beneficial to patients and their caregivers as they are provided with accurate and real time information for a quick response when the patient is in distress. The advantage that this novel system presents is based on the fact that it is inexpensive, uncomplicated, has a compact design, and is convenient and friendly in terms of usage. A significant number of health care systems that are used in monitoring patients' vital signs are available, but they are fraught with drawbacks such as being bulky, having a wired structure, and lacking precision.

The patient vital sign monitoring system presented in this article will automatically monitor body temperature, heart rate, and respiration rate. When a threshold is exceeded, an SMS, email, and an automated voice call will be sent to the caregiver. The automated voice call is important since SMS and email may not be read immediately by the caregiver and the patient may require urgent attention. The privacy of patients is guaranteed since caregivers have to biometrically verify themselves by either fingerprint or facial pattern to gain access to alerts. The device will be worn on a jacket and patients would be able to freely move around while their vital signs are automatically recorded and transmitted to a cloud data repository for analysis. Vital signs are the most basic functions of the body's measurements [10]. The four major vital signs that are usually monitored by medical professionals and other caregivers are body temperature, heart rate, respiration rate, and blood pressure which is not considered as a vital sign but is often measured alongside other vital signs. Vital signs are beneficial in the detection or observation of patients' medical problems; measurement of vital signs can be done in a medical environment, at home or elsewhere. Caregivers and even patients would be aware of critical conditions irrespective of the patient's location. In the development of the system, pulse sensor, thermometer sensor and respiration rate sensor are interfaced to an Arduino Uno microcontroller and the data are transmitted to the cloud data repository ThingSpeak. Caregivers are thus provided with timely and up to date alerts on the state of their patients. In validating the IoT-based patient vital signs monitoring system, three people had their body temperature, heart rate, and respiration rate respectively measured over a number of days. The accuracy of measurements taken using the prototype is confirmed by comparing with measurements taken simultaneously using stand-alone benchmark devices.

\section{BACKGROUND STUDY}

Due to the rapid advancement of wireless and web technologies specific to wearable monitoring devices, various mobile health applications have been reported to enhance healthcare monitoring. Deshkar et al. [11] undertook a comprehensive analysis of the operations and the underlying architecture of the current applications deployed. They discussed the major issues and challenges faced by these current applications. They established from their review that the IoT could provide health care-givers with vital data to improve on the outcome of patients; however, there are a number of challenges such as security concerns, privacy issues, inter-operability standards issues, legal, regulatory, and rights issues as well as emerging economy and development issues in the form of cost and speed of the internet. Their review confirms that the future lies with interconnected devices and how they are deployed and managed will determine how effective they may be. Similarly, S. Sengupta [12], a smart health application that uses an IoT-based infrastructure with authentication based on biometrics for monitoring and diagnosing the health condition of sick patients more effectively and efficiently was presented. Further, wearable wireless sensor devices is recommended by [12] due to patients' poor physical condition, as they transmit health-related parameters of the patients to the appropriate caregiver. The system ensures that one can only gain access to data after being biometrically authenticated which safeguards the privacy of patients' data. It can only be deployed in a hospital setting and is configured to grant access to physicians only and that is a major drawback since there are many patients 
who are at home. J. P. Rajan et al. [13] also presented a novel wireless architecture that monitors physiological signals in real time by virtual internet of things. They claimed their proposed system significantly improved the accuracy of remote monitoring compared to other wireless systems presented in the clinical domain. To gain access to a patient's data, one has to log into the system after being authenticated biometrically, this could waste time. An alert to the caregiver through a mobile device with details of the patient could speed up the process of attending to the patient.

Krishnan et al. [14] also presented an IoT-based health monitoring system for patients, measuring their hearth beat and temperature, they contend that patients are facing sudden death due to the absence of systems to monitor and alert them and their caregivers on their health situation. Further, Kumar and Rajasekaran et al. [15] also presented an IoT-based patient monitoring system that monitors the body temperature and respiratory rate of patients, they posit that the IoT presents the answer for effectively monitoring patients at a reduced cost. As a future work, they propose linking the MAC address of the Raspberry Pi and the programs to a website so that data could be stored and communicated to recipients. This confirms that after measurement of vital signs, the data cannot be communicated to the intended recipient which represents a major drawback of the system. R. N. Kirtana et al. [16], an IoT-based remote HRV Monitoring System which is user-friendly and inexpensive and used to monitor hypertensive patients on the borderline is proposed. In their estimation, monitoring of patients in real-time continues to be a critical challenge. When there is an emergency, a short message service (SMS) is sent to the care-taker and the doctor for immediate assistance. A major challenge is that SMS messages may not be seen by the care-taker or the doctor in time, a combination of SMS and automated voice call could be used. An IoT-based automated and intelligent system with the capability of sensing the health condition of a patient and is able to store and display the data gathered over the internet was presented by [16]. The doctor is provided with instant alerts when the condition of the patient is life-threatening. Their focus was to make their proposed system more user friendly and affordable. The proposed system's alert is limited to only when the patient is injured, it doesn't collect data on patients' vital signs. An Android platform based mobile application in the area of health care that combines the idea of IoT and cloud computing was also presented by [17]. Their application known as 'ECG Android App' affords the user the ability to visualise their electrocardiogram (ECG) and data are logged in the background and can be retrieved for analysis by medical personnel. The drawback of this system is that the medical personnel have to retrieve the record of the patient themselves since there is no automated response when a patient is in distress.

A monitoring system equipped with the capability of detecting a particular cardiac function abnormality with the ability to promptly measure a patient's temperature, heart rate and able to provide notification of an abnormal condition of a patient to a physician was presented in [18]. The physician has to manually request for data on the patient which is a major drawback of the system. A patient monitoring system for people affected by stroke that is capable of alerting doctors and caregivers about a patient's condition was also presented in [19]. The proposed model in diagnosing and predicting a patient's condition uses classification algorithms. This system does not use actual data from the patient but predicts the patient's condition and may be inaccurate. A. Rahman et al. [20], an intelligent patient monitoring system that automatically captures the health condition of a patient using various sensors and storing it on cloud locations was presented. Caregivers are able to monitor remotely the condition of a patient through a graphical information of a patient's condition. Where the condition is serious, an alert is sent to the doctor and the doctor or the patient has the ability to initiate a video call. The doctor may miss the alert since it is an SMS message, also where the condition of the patient is very critical he/she may not be able to respond to a video call and that could be life-threatening. A patient monitoring system that monitors two main vital signs: body temperature and respiratory rate implemented in an IoT platform is presented in [21]. Its purpose is to detect in real time, vital sign levels of patients and analyse the data according to the patient's age and alert caregivers of any problem. Only temperature and respiratory rate, two key vital signs, are measured, also the results require more accuracy as the authors have recommended a higher sensitivity sensor as a future work to ensure more precise results. A framework that regularly records body temperature, heart rate, and observation of ECG of patients using sensors; the data are transmitted to a cloud repository which is sent to a doctor or a nurse for urgent attention where required was presented by [22]. They concede that the security of their system requires improvement as the use of HTTPS is insufficient to protect their system from data sniffing, also fake data could also be sent to servers due to poor data authorisation techniques.

J. Gomez et al. [23], an ontology-based architecture that is able to monitor the health and routine workout recommended for patients who have chronic diseases was proposed. The proposed system monitors diabetes and heart rate, it also directs patients to improve their eating habits and workout routines. This has not been practically implemented and may not be feasible. A smart patient monitoring system for the automatic observation of the biological behaviour of patients which is then forwarded to a cloud repository for analysis and reporting was presented in [24]. Caregivers are provided with up-to-date information on their 
patients for action where necessary. This system as confirmed by the authors in their recommendation for future work is not wearable and so does not monitor patients continuously. It doesn't also monitor babies and older people from any location. A Deep Neural Network based smart patient monitoring system designed with smart sensors was also presented in [25]. In their estimation, the presented system's energy consumption is low and is more accurate in predicting a patient's vital sign. This system does not gather actual data in real time, it is based on predictions which could be inaccurate.

In summary, all the literature perused agree that automatically monitoring patients' vital signs both at the hospital, home, and at any location they may find themselves is very vital. This will promote the health of patients and ensure that quick help is offered these patients when they are in distress. The measurement of the vital signs of patients must be accurate; an erroneous reading can delay help to the patient which could be fatal. Most of the proposed systems measure only one or two vital signs. All the systems presented and analysed in the literature perused transmit alerts to caregivers through SMS which could be missed since SMS alerts come with a short beep or no alert at all. In other proposed systems, physicians and other caregivers have to log into a data repository in order to gain access to patients' data to know their condition. A system that alerts caregivers when patients' vital signs data exceeds the normal threshold by placing a continuous voice call until the caregiver picks in addition SMS message is desired to save patients' lives. It must be accurate and wearable so that the patient can use it 24 hours irrespective of his/her location.

\section{RESEARCH METHOD}

The theoretical framework underlying the proposed IoT based patient vital signs monitoring system and its architecture is presented in this section.

\subsection{Theoretical foundation}

In this section, a theoretical framework for the internet of things Application in patient vital signs monitoring is presented. Figure 1 represents the complete framework that links the concepts associated with the proposed patient vital signs measuring system. The framework is made up of 4 sub-components which are the person, network of sensors, input and storage, and data processing, analysis, and output.
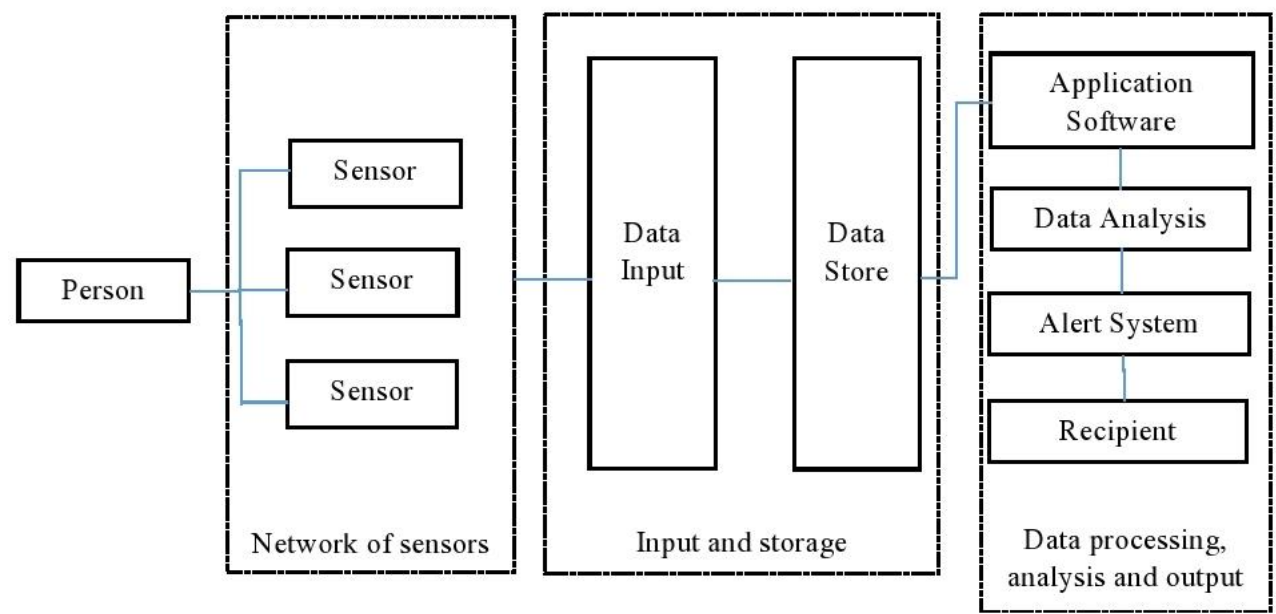

Figure 1. Theoretical framework of IoT based vitals monitoring system

From Figure 1, the Person denotes the patient who is the main character in the proposed system. The network of sensors are the sensors that are used to measure the vital signs of the patient. In the input and storage component, a microcontroller is used to gather the input from the sensors and the data is transmitted to a cloud-based data repository for storage. Using an Application Software, the data is retrieved from the data storage and is subjected to analysis. The application software should automatically read data anytime the data repository is updated with new data for analysis. Where a threshold is surpassed after analysis, an alert is to be triggered to a recipient who in this case is a caregiver. The form of the alert continues to be a problem for researchers, most of whom have recommended SMS when the recipient is in a remote location and an alarm when the recipient is in the same premise as the patient. For novelty, this framework uses SMS, email, 
and a continuous automated phone call to the caregiver with a voice message detailing the patients name and details of the condition necessitating the call. To overcome the issue of privacy where anyone can get access to the call, each caregiver is made to biometrically register for authentication. It is only by biometric authentication that the caregiver can be granted access to the incoming alert. This will ensure that the security of the system is guaranteed and privacy of patients is safeguarded.

\subsection{Architecture of the proposed system}

Based on the theoretical framework, the architecture of the IoT based patient vital signs monitoring system is designed. The design is expected to produce an inexpensive, portable and easy to use wearable system capable of gathering data accurately in real time and transmit to a caregiver securely for prompt response. The outline of the proposed system architecture is depicted in Figure 2. Three sensors, namely: pulse sensor, temperature sensor, and respiration rate sensor are used to collect data on vital signs automatically from the patient through an Arduino Microcontroller.

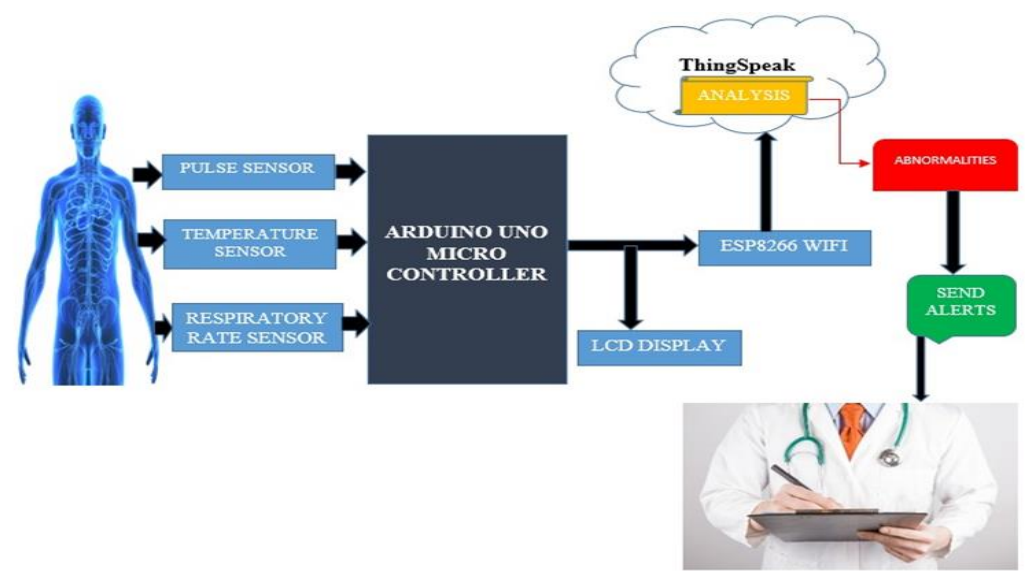

Figure 2. Architecture of the proposed system

The collected data are displayed on the LCD display unit, and through the Wi-Fi ESP 8266 Module the data are transmitted to the ThingSpeak cloud data repository for analysis. After automatically analyzing the data, a short message service (SMS) alert and a voice message through a phone call is sent to caregivers where an urgent attention is required to save the patient from an imminent danger. A schematic diagram of the proposed system is shown in Figure 3.

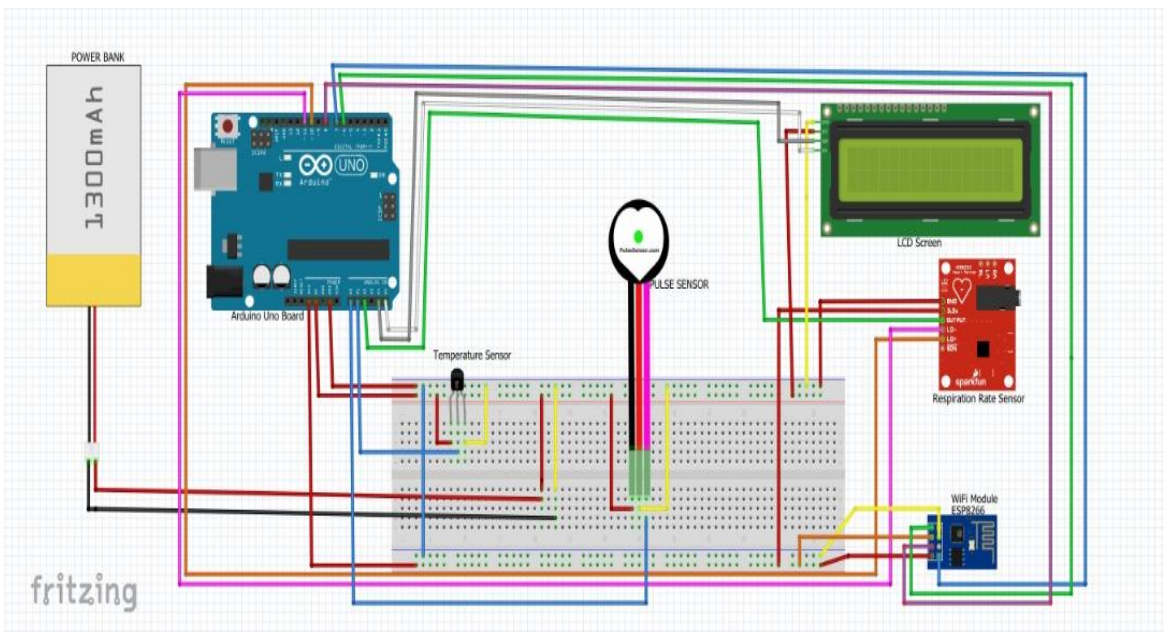

Figure 3. The schematic view of the proposed system 
The Arduino Uno as shown in Figure 3 is a microcontroller module based on ATmega 328P. It is comprised of 14 pins ( 6 of them for PWN outputs), 6 analog inputs, 16 quartz crystals (16 MHz), USB, power jack and reset button. It has the option for the addition of a USB connection. An LM35 temperature sensor that will ensure the gathering of data on a patient's temperature is connected to the microcontroller. The LM35 temperature sensor is a device specifically designed to determine an object's level of hotness or coldness. LM35 is an integrated circuit temperature sensor that is precise, its output voltage fluctuates depending on the temperature around it. It is a miniaturized and inexpensive IC which is capable of measuring temperature ranging from $-55^{\circ} \mathrm{C}$ to $150^{\circ} \mathrm{C}$ which makes it ideal for use in this project to measure body temperature. It can be interfaced to any microcontroller such as Arduino Uno. As shown in Figure 3, the IC can be powered through the application of a controlled voltage like $+5 \mathrm{~V}$ (VS) to the input pin and the ground pin is connected to the ground of the circuit which then allows the measurement of the temperature in the form of a voltage. A pulse sensor that measures patient heart rate is also connected to the microcontroller as shown in Figure 3. The pulse sensor has a simplistic working principle, it has two interfaces, on the first interface, the light-emitting diode and ambient light sensor is connected. When the blood flow is sensed then the ambient light sensor will receive more light as they will be reproduced by the flow of blood.

A pulse wave is the increase in blood vessel volume that happens as the cardiac pumps blood and a detection or measurement of this increase is done by the pulse sensor. A respiration rate sensor, ADS1292R ECG/Respiration shield for Arduino that measures the number of breaths of a patient within a specified period is also connected to the microcontroller as shown in Figure 3. The sensor measures respiration by using impedance pneumography, through a change in chest impedance. It has $3.3 \mathrm{~V}$ on board low noise voltage regulator and a logic level on-board translator for interfacing to Arduino. The output of the sensor is processed in Arduino Uno microcontroller such that the real-time respiratory rate (breath per minute) can be gathered to be saved subsequently. To transmit the data to a cloud data repository through the internet, a WiFI Module, the ESP8266 Wi-Fi Module is used. The ESP8266 Wi-Fi module is easy to use and low-cost device that provides internet connectivity for the proposed system. This module can act both as an access point which is capable of being used as a hotspot and a station, it can be linked with Wi-Fi providing the Internet of Things with the simplest possible way of collecting and uploading data. It can also download data from the internet by means of APIs, so the project can access any available information on the internet, making them smarter. The module can also be programmed with the Arduino IDE which makes it even more user-friendly. The module has only 2 GPIO pins and so can be used along with a microcontroller, such as Arduino. Its features include: Low cost, compact and powerful Wi-Fi Module, Power supply: $+3.3 \mathrm{~V}$ only, current consumption: $100 \mathrm{~mA}$, it can be used as a station or Access Point or both combined. To display the data on a screen during the data gathering process, a 16x2 LCD Display is connected to the microcontroller. The $16 \times 2$ translate a display of 16 characters per line in 2 such lines. In this LCD each character is displayed in a $5 \times 7$-pixel matrix.

\subsection{Building the IoT-based patient monitor}

The arctitecture of the IoT based patient vitals monitor is converted into a prototype by connecting the various sensors to the Arduino uno microcontroller and configured to establish connectivition to the internet for data transmission to the cloud. The equipment used in building the system are inexpensive and robust which is a requirement in our quest to collect accurate data and also avoiding a break down. The process of connecting the devices is enumerated below:

a) Connecting the LCD screen to the arduino uno microcontroller board

The Arduino Uno Microcontroller Board has a USB port that allows users to connect to a computer device in other to upload verified codes to it. First, the LCD is placed on the Serial I2C board to convert and make the number of cables to be plugged on the Arduino Board much smaller. The I2C adapter have 4 pins which are VCC, GND, SDA and SCL. First GND and 5V power are connected from the Arduino Board to the breadboard and as such providing Power and Ground for all connections whilst using just one 5V pin on the Arduino Board as well as 1 GND pin. The GND pin is connected to the GND slot in bread board which have been connected to the Arduino Board, VCC pin is connected to the $5 \mathrm{~V}$ on the breadboard, SDA is connected to the A4 on the Arduino Uno Board and SCL is connected to the A5 on the Arduino Uno Board.

b) Connecting the LM35 temperature sensor to the arduino uno microcontroller board

The LM35 Sensor is very accurate in attaining the temperature of anything that attached to it hence making it very ideal to read the patient's body temperature. It has 3 Pins which are the VCC, GND and OUT/Signal Pin. The VCC and GND are both connected to the 5V and GND on the breadboard respectfully. The OUT/Signal Pin is connected to the AO on the Arduino Board. Figure 3 shows Arduino microcontroller integrated with LCD and the LM35 sensor. 
c) Connecting the pulse sensor SEN-11574 to the arduino uno microcontroller board

In order for the Pulse sensor to operate, connect the 3 pins which are the GND, VCC, and the signal pin. The GND pin and VCC pin are connected to the GND and VCC on the breadboard. The signal pin is connected to the A1 on the Arduino Board.

d) Connecting the ADS1292R ECG/respiration shield for arduino

To connect the ADS1292R ECG/Respiration shield to the Arduino board, pin VDD is connected to the $+5 \mathrm{~V}$ to supply power, GND is also connected to Gnd, START is connected to D5, DRDY (data ready) is connected to D6, MOSI is connected to D11 (slave in), MISO is connected to D12 (Slave out). The resultant prototype of the IoT based Patient Monitoring system is depicted in Figure 4.

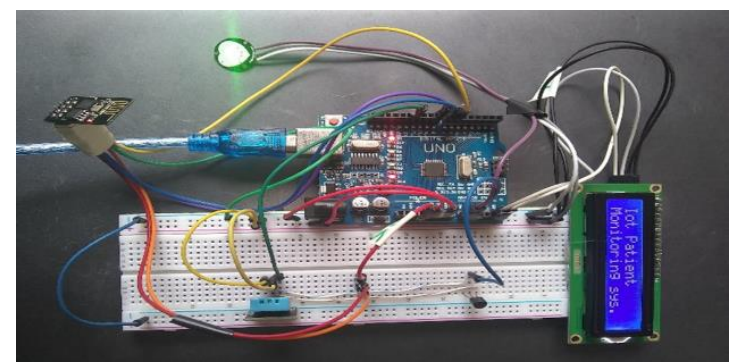

Figure 4. Prototype of the IoT based patient monitoring system

e) Powering the prototype

To ensure the monitoring of patients' condition at all times irrespective of where they might find themselves, the device is powered from a $5 \mathrm{~V}$ lithium power bank connected to the Arduino board through a USB. This will enable it to be worn using a jacket. It will result in the device being small and light and also will have enough power to last long. The device will report with a beep and on the LCD when power is left with about $10 \%$ to run out. There will always be an additional power bank that will replace the one that is running out.

\subsection{Transmitting alerts to caregivers}

To safeguard the privacy of patients, caregivers are registered biometrically onto the system by capturing their fingerprints and facial patterns. The data is stored in a database; each patient is assigned a number of caregivers who are listed in order. This is to ensure that other caregivers can be contacted in the event that the first person is not available. When a patient is in distress, an alert in the form of SMS and email specifying the details of the patient's condition is sent to the caregiver. A time threshold is established and on the expiry of the threshold, when the message is not viewed by the caregiver, a voice call is continuously placed to the caregiver. Alerts through voice calls could be received faster than SMS and email. To access the SMS, email, and the voice call, a caregiver has to be authenticated by fingerprint or facial pattern. The caregiver after being successfully authenticated gains access to alert and hurriedly attends to the patient.

\section{RESULTS AND DISCUSSION}

A prototype of the Patient Monitoring System is shown in Figure 4. This system has been developed with the capability of tracking patient heart rate, body temperature, and respiratory rate. The system transmits the readings in real time over to the cloud database platform, ThingSpeak, for remote monitoring by authorized persons. It is expedient to test the accuracy and speed with which vital signs are measured. The prototype is worn in a jacket by a person whose temperature is monitored for three days in an interval of one hour; the measurement is taken automatically eight (8) times a day. A normal body temperature could vary from person to person due to age, activity and time of the day. It is believed that normal temperature of the body is between $36.1^{\circ} \mathrm{C}$ and $37.2^{\circ} \mathrm{C}$, in analyzing the data in ThingSpeak cloud data repository, a threshold temperature of $38^{\circ} \mathrm{C}$ and above is set as critical requiring urgent attention. When such a critical stage is reached, the system automatically sends an SMS alert to a caregiver's mobile device for urgent response. The care giver is able to view the data after a biometric a biometric authentication. In testing the prototype, the temperature of a person is taken automatically using the IoT based patient vital signs monitoring system, the data are stored in a cloud data repository and it is presented in a graphical form as shown in Figure 5.

A normal heart rate for adult's ranges from 60 to 100 beats per minute. The IoT Based Patient Monitoring System is used to measure the heart rate of a patient twice a day at $7 \mathrm{am}$ and $7 \mathrm{pm}$ for eight (8) 
days which is eight times. Figure 6 shown depicts the heart rate measurement of a patient. A threshold of 101 beats per minute is set; when the data are sent to the cloud repository, it is analyzed and where the value transmitted is more than the repository, an alert is immediately sent to a caregiver to respond immediat

The next patient vital sign monitored is the respiration rate. A normal respiration rates for an adult person at rest ranges from 12 to 16 breaths per minute, a threshold of 18 breaths per minute is set. The IoT Based Patient Monitoring System is used to monitor the respiration of a patient twice a day at $8 \mathrm{am}$ and $5 \mathrm{pm}$ for eight (8) days, i.e., eight times. A graphical display of the respiration rate measurement of a person taken for 8 days is depicted in Figure 7.

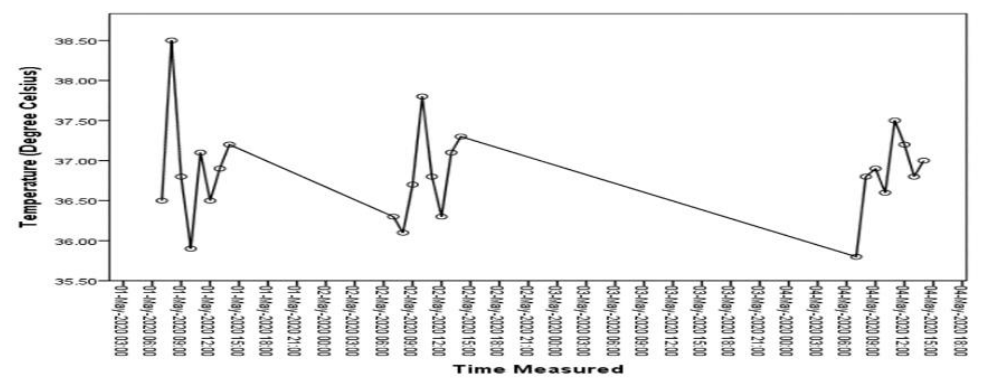

Figure 5. Temperature measurement of a patient

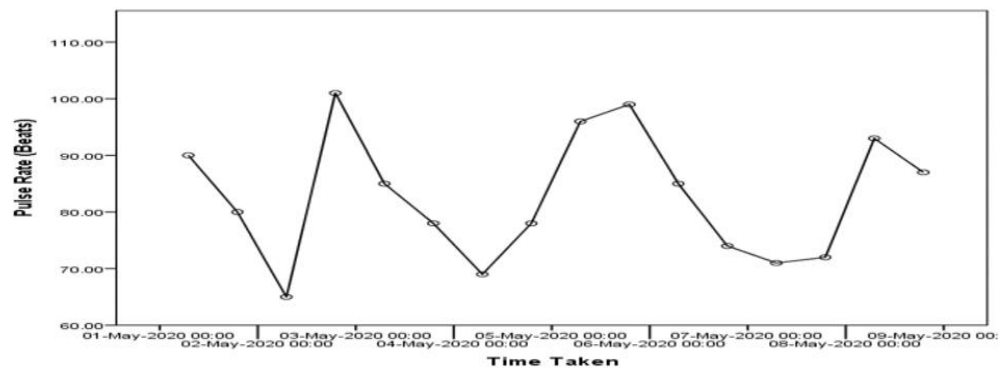

Figure 6. Heart rate measurement of a patient

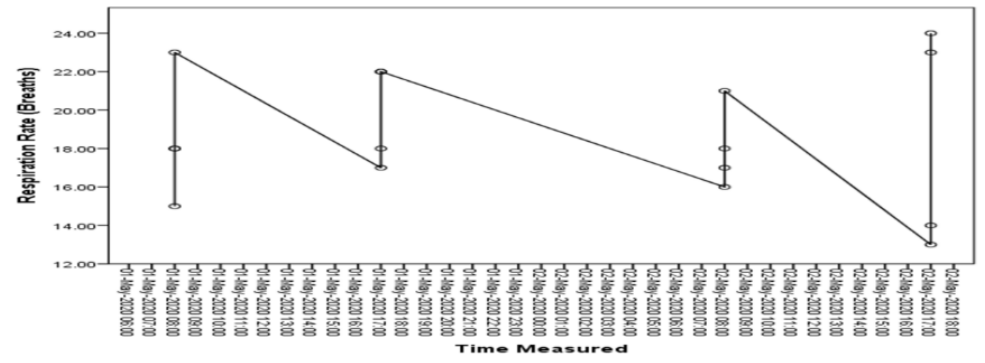

Figure 7. Respiration rate measurement of a patient

\subsection{Validation of the proposed}

The proposed patient vitals measuring system should accurately measure temperature, heart rate, and respiratory rate of patients within the hospital or wherever they find themselves accurately. Where an abnormality is detected, an automatic SMS, Email and automated voice call should be sent to the medical doctor or caregiver immediately. Security and privacy should be guaranteed; to gain access, the caregiver must be biometrically authenticated. To confirm the novelty of the proposed IoT based Patient Monitoring System, it is compared against other benchmark systems presented earlier and discussed in 2 which is shown in Table 1.

From Table 1, it is evident that most of the proposed systems measure only 2 vital signs while a few others measure only 1 vital sign. None of the systems proposed measure temperature, heart rate, and respiratory rate at the same time. During emergency, only [19] use both SMS and email to alert caregivers, 
others use either SMS or email. Caregivers may not check their emails and SMS messages frequently and that could put the lives of patients who require immediate assistance in danger. The proposed system sends an SMS alert and email to a caregiver when a patient is in distress, however, it is very likely the caregiver may not check for an SMS or email on time. A telephone call with an automated voice containing details of the patient and his/her condition is placed to the caregiver. The device's alarm sounds until the caregiver picks and listens to the voice message which can be repeated after selecting an option. This will ensure that the patient receives prompt attention. In terms of authentication to ensure the privacy of patients, only [12] uses biometric authentication but the drawback of the system is that to gain access to the data of a patient, the caregiver has to $\log$ into the system using an android application. The proposed system is novel, it measures 3 vital signs at the same time i.e., temperature, respiration rate, and heart rate. It also transmits alerts to a caregiver using SMS, email and automate voice call. The proposed system also ensures the privacy of individuals by ensuring that caregivers are authenticated biometrically on their mobile devices before they gain access to patients' data. To ensure the security of the system, 3 types of biometric authentications are provided namely; fingerprint, facial, and iris. A caregiver can use any of them to authenticate him/her self to gain access to the alert. Measuring the 3 vital signs, and transmitting the alert through SMS, email, and voice with biometric authentications makes the proposed system unique and novel.

Table 1. Comparison with benchmark systems

\begin{tabular}{|c|c|c|c|c|c|c|c|}
\hline \multirow[b]{2}{*}{ System } & \multicolumn{3}{|c|}{ Vital Signs } & \multicolumn{3}{|c|}{ Mode of Alert } & \multirow{2}{*}{$\begin{array}{c}\text { Biometric } \\
\text { Authentication }\end{array}$} \\
\hline & Temperature & Heart Rate & $\begin{array}{l}\text { Respiratory } \\
\text { Rate }\end{array}$ & SMS & Email & Phone Call & \\
\hline Ani et al. [19] & No & Yes & No & Yes & Yes & No & No \\
\hline Hadis et al. [21] & Yes & No & Yes & No & No & No & No \\
\hline Kirtana \& Lokeswari [16] & No & Yes & No & Yes & No & No & No \\
\hline Krishnan et al. [12][14] & Yes & Yes & No & No & No & No & No \\
\hline Mohammed et al. [17] & No & Yes & No & No & No & No & No \\
\hline Sengupta [12] & Yes & Yes & No & No & No & No & Yes \\
\hline $\begin{array}{l}\text { Proposed IoT based Patient } \\
\text { Monitoring System }\end{array}$ & Yes & Yes & Yes & Yes & Yes & Yes & Yes \\
\hline
\end{tabular}

\subsection{Confirming measurement accuracy}

To confirm the accuracy of the IoT based patient monitoring system, the prototype is used to measure temprature, pulse rate, and respiration rate of fifteen (15) people. Simultaneously, 3 widely used and trusted adevices namely; Braun Forehead Thermometer, Prove Fingertip Pulse Oximeter, Neulog Respiration Belt Monitor were also used to measure the temperature, pulse and respiration rate of the same people respectively. The measured values from the prototype and the benchmark devices are analysed and presented in Figure 8. As shown in Figure 8, the measurements attained by the prototype is near the measurement attained by the benchmark stand-alone devices thereby confirming the accuracy of the prototype.

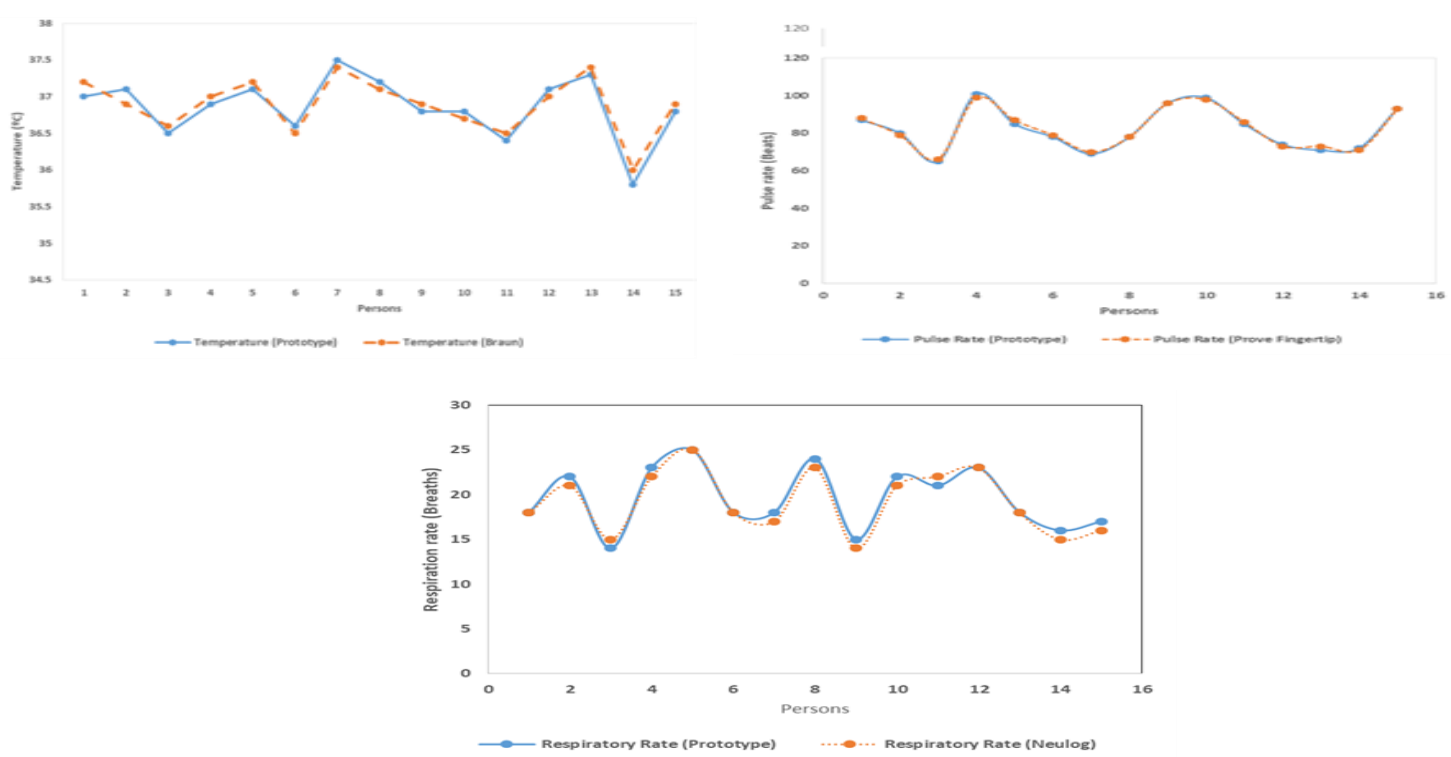

Figure 8. Comparison of measurements by prototype against benchmark devices 


\section{CONCLUSION}

The domain of health care has been greatly enhanced with the introduction of the IoT. This has enabled the monitoring remotely of the condition of patients. Vital signs which are the measurement of the basic functions of the body are monitored routinely by patients' caregivers so as to make sure that any emergency can be easily tracked for prompt action. In this paper, an IoT Based Patients' Vital Signs monitoring system that accurately measures patients' body temperature, pulse rate, and respiration rate is presented. This will ensure that patients whether at home or in the hospital can be monitored and attended to when they require immediate attention. It will save the lives of those who otherwise would have perished due to lack of immediate attention. Remote monitoring systems that have been presented to be used in patient management are bedevilled with various problems such as power consumption, bulkiness, inefficiency in data exchange, and ability to monitor more than one vital sign. The prototype was subjected to performance testing for a number of days, and found able to record and transmit accurate data on the vital signs of a patient to a cloud data repository for storage and analysis. The system was able to measure in real time accurate abnormal vital sign values culminating in an SMS alert, email, and a voice call sent to a caregiver. Caregivers only get access to the emergency message received after being verified using either of 2 biometric authentications, namely; facial and fingerprint. The novelty of the proposed system is its ability to monitor accurately three vital signs at the same time; transmit the data by SMS, email, and voice. The user is also authenticated biometrically to ensure the privacy of patients. As a future work, the system shall be modified to include a real time blood pressure monitor.

\section{REFERENCES}

[1] D. Stuckler, "Population Causes and Consequences of Leading Chronic Diseases: A Comparative Analysis of Prevailing Explanations," The Milbank Quarterly, vol. 86, no. 2, pp. 273-326, 2008, doi: 10.1111/j.14680009.2008.00522.x.

[2] P. A. Laplante, M. Kassab, N. L. Laplante, and J. M. Voas, "Building Caring Healthcare Systems in the Internet of Things,” IEEE Systems Journal, vol. 12, no. 3, pp. 3030-3037, Sept. 2018, doi: 10.1109/JSYST.2017.2662602.

[3] D. Miorandi, S. Sicari, F. D. Pellegrini, and I. Chlamtac, "Internet of things: Vision, applications and research challenges," Ad Hoc Networks, vol. 10, no. 7, pp. 1497-1516, 2012, doi: https: 10.1016/j.adhoc.2012.02.016.

[4] Z. U. Ahmed, M. G. Mortuza, M. J. Uddin, M. H. Kabir, M. Mahiuddin, and M. J. Hoque, "Internet of Things Based Patient Health Monitoring System Using Wearable Biomedical Device," International Conference on Innovation in Engineering and Technology ICIET, 2018, pp. 1-5, doi: 10.1109/CIET.2018.8660846.

[5] M. Neyja, S. Mumtaz, K. M. S. Huq, S. A. Busari, J. Rodriguez, and Z. Zhou, "An IoT-Based E-Health Monitoring System Using ECG Signal," GLOBECOM 2017-2017 IEEE Global Communications Conference, 2017, pp. 1-6, doi: 10.1109/GLOCOM.2017.8255023.

[6] Z. Wang, Z. Yang, and T. Dong, "A Review of Wearable Technologies for Elderly Care that Can Accurately Track Indoor Position, Recognize Physical Activities and Monitor Vital Signs in Real Time,” Sensors, vol. 17, no. 2, p. 341, 2017, doi: 10.3390/s17020341.

[7] R. Iyer and R. Mishra, "Building Intelligent Internet of Things Applications using Microsoft Stream Insight," CSI Communications, 2012.

[8] T. J. McCue, "\$117 Billion Market For Internet of Things In Healthcare," Forbes, 2020. [Online]. Available: https://www.forbes.com/sites/tjmccue/2015/04/22/117-billion-market-for-internet-of-things-in-healthcare-by-2020/.

[9] P. Singh, "Internet of Things Based Health Monitoring System: Opportunities and Challenges," Int. J. of Advanced Research in Computer Science, vol. 9, no. 1, pp. 224-228, 2018, doi: 10.26483/ijarcs.v9i1.5308.

[10] M. J. M. Breteler, et al., "Vital Signs Monitoring with Wearable Sensors in High-risk Surgical Patients," Anesthesiology, vol. 132, no. 3, pp. 424-439, 2020, doi: 10.1097/ALN.0000000000003029.

[11] S. Deshkar, R. A. Thanseeh, and V. G. Menon, "A review on IoT based m-Health systems for diabetes," International Journal of Computer Science and Telecommunications, vol. 8, no. 1, pp. 13-18, 2017.

[12] S. Sengupta, "A Secured Biometric-Based Authentication Scheme in IoT-Based Patient Monitoring System," Advances in Intelligent Systems and Computing Emerging Technology in Modelling and Graphics, pp. 501-518, 2019, doi: 10.1007/978-981-13-7403-6_44.

[13] J. P. Rajan and S. E. Rajan, "An Internet of Things based physiological signal monitoring and receiving system for virtual enhanced health care network," Technology and Health Care, vol. 26, no. 2, pp. 379-385, 2018, doi: 10.3233/THC-171173.

[14] D. S. R. Krishnan, S. C. Gupta, and T. Choudhury, "An IoT based Patient Health Monitoring System," International Conference on Advances in Computing and Communication Engineering ICACCE, 2018, pp. 1-7, doi: 10.1109/ICACCE.2018.8441708.

[15] R. Kumar and M. P. Rajasekaran, "An IoT based patient monitoring system using raspberry Pi," International Conference on Computing Technologies and Intelligent Data Engineering ICCTIDE'16, 2016, pp. 1-4, doi: 10.1109/ICCTIDE.2016.7725378.

[16] R. N. Kirtana and Y. V. Lokeswari, "An IoT based remote HRV monitoring system for hypertensive patients," International Conference on Computer, Communication and Signal Processing ICCCSP, 2017, pp. 1-6, doi: 10.1109/ICCCSP.2017.7944086. 
[17] J. Mohammed, C. Lung, A. Ocneanu, A. Thakral, C. Jones, and A. Adler, "Internet of Things: Remote Patient Monitoring Using Web Services and Cloud Computing," IEEE International Conference on Internet of Things (iThings), 2014, pp. 256-263, doi: 10.1109/iThings.2014.45.

[18] A. Julius and Z. Jian-Min, "IoT Based Patient Health Monitoring System Using LabVIEW and Wireless Sensor Network," Int. J. of Science and Research, vol. 6, no. 3, pp. 894-900, 2017, doi: 10.21275/ART20171643.

[19] R. Ani, S. Krishna, N. Anju, M. S. Aslam, and O. S. Deepa, "Iot based patient monitoring and diagnostic prediction tool using ensemble classifier," International Conference on Advances in Computing, Communications and Informatics ICACCI, 2017, doi: 10.1109/ICACCI.2017.8126068.

[20] A. Rahman, T. Rahman, N. H. Ghani, S. Hossain, and J. Uddin, "IoT Based Patient Monitoring System Using ECG Sensor," International Conference on Robotics, Electrical and Signal Processing Techniques ICREST, 2019, pp. 378-382, doi: 10.1109/ICREST.2019.8644065.

[21] N. S. M. Hadis, M. N. Amirnazarullah, M. M. Jafri, and S. Abdullah, "IoT Based Patient Monitoring System using Sensors to Detect, Analyse and Monitor Two Primary Vital Signs," Journal of Physics: Conference Series, 2020, vol. 1535 , no. 1 , p. 012004.

[22] L. N. Omran, K. A. Ezzat, A. Bayoumi, A. Darwich, and A. E. Hassanien, "IoT-based intensive care secure framework for patient monitoring and tracking," International Journal of Grid and Utility Computing, vol. 10, no. 5, pp. 475-487, 2019, doi: 10.1504/IJGUC.2019.102017.

[23] J. Gómez, B. Oviedo, and E. Zhuma, "Patient Monitoring System Based on Internet of Things," Procedia Computer Science, vol. 83, pp. 90-97, 2016, doi: 10.1016/j.procs.2016.04.103.

[24] M. S. Uddin, J. B. Alam, and S. Banu, "Real time patient monitoring system based on Internet of Things," 4th Int. Conf. on Advances in Electrical Engineering ICAEE, 2017, pp. 516-521, doi: 10.1109/ICAEE.2017.8255410.

[25] P. R. Jeyaraj and E. R. S. Nadar, "Smart-Monitor: Patient Monitoring System for IoT-Based Healthcare System Using Deep Learning," IETE Journal of Research, pp. 1-8, 2019, doi: 10.1080/03772063.2019.1649215.

\section{BIOGRAPHIES OF AUTHORS}

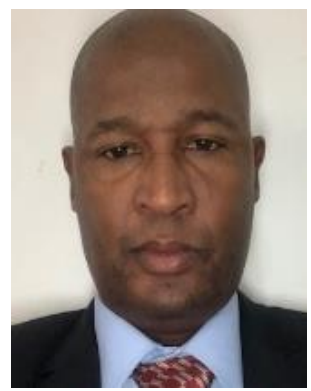

Osman Yakubu was born in Kumasi, Ghana, he obtained a BSc. Computer Science degree from the Kwame Nkrumah University of Science and Technology, Kumasi, Ghana in 2005, MSc. Informatics from the University of Ulster, Belfast, UK in 2007, and a PhD in Computer Science from M. S. Ramaiah University of Applied Sciences, Bangalore, India in 2020. He also obtained a certificate in 'Enabling Technologies for the Internet of Things' at the University of Pisa, Italy in 2017 and certificate in 'Quantitative Data Analysis in R' at the Radboud University, Nijmegen, Netherlands in 2019. His Research Interests are internet of things, Data Science, and Biometric Systems. Currently lecturing and offering IT consultancy services.

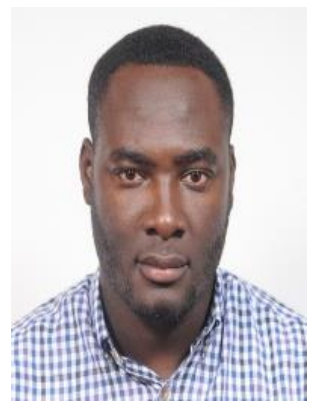

Emmanuel Wereko was born in Kumasi, Ghana. He attended Garden City University College in Kumasi, Ghana an graduated in 2020 with a BSc. Degree in Computer Science. His research interests are Internet of Things, data science and data mining. 